Pacific Journal of Mathematics

INVOLUTIONS FIXING CODIMENSION TWO KNOTS 


\section{INVOLUTIONS FIXING CODIMENSION TWO KNOTS}

\section{ChaO-Chu Liang}

1. Involution. An $m$-knot $\left(\Sigma^{m+2}, M^{m}\right)$ consists of an $(m+2)$ homotopy sphere $\Sigma^{m+2}$ and an $m$-homotopy sphere $M^{m}$ differentiably (or piecewise linearly) embedded in it. A $(2 n-1)$-knot is callsd simple if $\pi_{j}(\Sigma-M)=\pi_{j}\left(S^{1}\right)$ for $j<n$. It is well known that each knot cobordism class contains a simple knot, [5] or [7].

Associated to each $(2 n-1)$-knot, we have Seifert matrices $B$, with $B+\varepsilon B^{\prime}$ unimodular, where $\varepsilon=(-1)^{n}$ and $B^{\prime}$ denotes the transpose of $B$. For $n \geqq 2$, the isotopy class of simple knot is completely determined by its Seifert matrices [8].

In $[1, \S 11]$, Cappell and Shaneson used their algebraic $K$-theoretic obstruction groups to determine which knot cobordism classes admit semifree $Z_{p}$ actions fixing the knots. In $\S 3$ below, we will prove the following theorem from the viewpoint of [5] and [8].

Theorem 1. A simple knot $\left(\Sigma^{2 n+1}, M^{2 n-1}\right), n \geqq 3$, admits a p. 1 . involution $T$ fixing $M^{2 n-1}$ if and only if it has an associated Seifert matrix $B$ of the form $B=A\left(A-\varepsilon A^{\prime}\right)^{-1} A$ for some matrix $A$ with both $A+\varepsilon A^{\prime}$ and $A-\varepsilon A^{\prime}$ being unimodular.

We will also discuss the differentiable case in the last section.

2. A technical lemma. Recall that $\varepsilon=(-1)^{n}$.

LemMa 2. Let $A$ be an $(r \times r)$-matrix with both $A+\varepsilon A^{\prime}$ and $A-\varepsilon A^{\prime}$ being unimodular. Then the following system of equations has a unique solution for the pair of $(r \times r)$-matrices $C_{1}$ and $C_{2}$.

$$
\begin{gathered}
C_{1} A+\varepsilon C_{2} A^{\prime}=A+\varepsilon A^{\prime} \\
\varepsilon C_{1} A^{\prime}+C_{2} A=0 .
\end{gathered}
$$

Proof.

$$
(1)+(2) C_{1}\left(A+\varepsilon A^{\prime}\right)+C_{2}\left(A+\varepsilon A^{\prime}\right)=A+\varepsilon A^{\prime} .
$$

Since $A+\varepsilon A^{\prime}$ is unimodular, (3) becomes

$$
C_{1}+C_{2}=I \text {, the identity }
$$

$$
\text { (1) }-(2) C_{1}\left(A-\varepsilon A^{\prime}\right)-C_{2}\left(A-\varepsilon A^{\prime}\right)=A+\varepsilon A^{\prime} \text {. }
$$

Since $A-\varepsilon A^{\prime}$ is unimodular, (5) becomes 


$$
C_{1}-C_{2}=\left(A+\varepsilon A^{\prime}\right)\left(A-\varepsilon A^{\prime}\right)^{-1} .
$$

From (4) and (6), we have

$$
C_{1}=A\left(A-\varepsilon A^{\prime}\right)^{-1} \text { and } C_{2}=-\varepsilon A^{\prime}\left(A-\varepsilon A^{\prime}\right)^{-1} .
$$

3. Proof of Theorem 1. If a simple $\operatorname{knot}\left(\sum^{2 n+1}, M^{2 n-1}\right), n \geqq 3$, admits a $p .1$. (or differentiable) involution $T$ fixing $M^{2 n-1}$, then it is easy to see that $\Sigma_{1}=\Sigma / T$ is a $(2 n+1)$-homotopy sphere, and $\left(\Sigma_{1}, M\right)$ is again a simple knot. Let $Y$ be the closure of $\left(\Sigma_{1}-M \times D^{2}\right)$, and $V^{2 n} \subseteq Y$ be an $(n-1)$-connected Seifert manifold for $\left(\Sigma_{1}, M\right)$ with $\partial V=M \times e^{i 0}$, (we consider $S^{1}=\left\{e^{i \theta}\right\}$ ), [5], [7]. Lifting $V$ to $\Sigma$, we have two equivariant Seifert manifolds $V_{1}$ and $V_{2}$ with $T V_{1}=$ $V_{2}, \partial V_{1}=M \times e^{i 0}$, and $\partial V_{2}=M \times e^{i \pi}$, [9]. We then cut $X=$ closure of $\left(\Sigma-M \times D^{2}\right)$ along $V_{1}$ to get a manifold $W$.

$(*)$

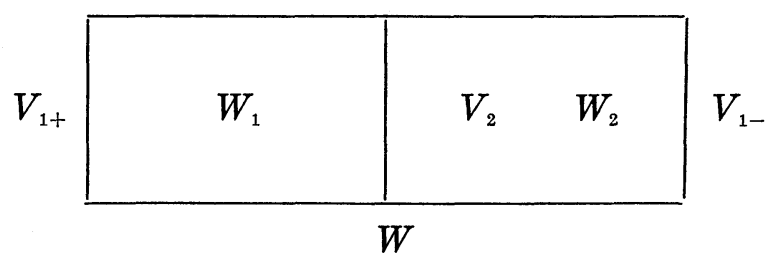

We see immediately that $W_{1}$ is the manifold obtained from $Y$ (in $\Sigma_{1}$ ) by cutting it along $V$ and $W_{2}=T W_{1}$. Let $\left\{e_{1}, \cdots, e_{r}\right\}$ be a basis for $H_{n} V_{1+}$, and $\left\{f_{1}, \cdots, f_{r}\right\}$ a basis for $H_{n} W$ determined by the Alexander duality (in $\Sigma$ ). Similarly, viewing $\left\{e_{i}\right\}$ as a basis for $H_{n} V$, we have a basis $\left\{d_{i}\right\}$ for $H_{n} W_{1}$ by using the Alexander duality in $\Sigma_{1}$. Let $A$ and $B$ be the Seifert matrices associated to $\left(\Sigma_{1}, M\right)$ and $(\Sigma, M)$ respectively (with respect to the basis $\left\{e_{i}\right\}$ ) [5], [7].

From [5], we know that $A$ represents the map $H_{n} V_{1+} \rightarrow H_{n} W_{1}$ with respect to the bases $\left\{e_{i}\right\}$ and $\left\{d_{i}\right\}$, also the map $H_{n} V_{2} \rightarrow H_{n} W_{2}$ with respect to the bases $\left\{T_{*} e_{i}\right\}$ and $\left\{T_{*} d_{i}\right\}$. The matrix $-\varepsilon A^{\prime}$ represents the map $H_{n} V_{2} \rightarrow H_{n} W_{1}$ with respect to the bases $\left\{T_{*} e_{i}\right\}$ and $\left\{d_{i}\right\}$, also the map $H_{n} V_{1-} \rightarrow H_{n} W_{2}$ with respect to the bases $\left\{e_{i}\right\}$ and $\left\{T_{*} d_{i}\right\}$. The matrix $B$ represents $H_{n} V_{1+} \rightarrow H_{n} W$ with respect to the bases $\left\{e_{i}\right\}$ and $\left\{f_{i}\right\}$, and $-\varepsilon B^{\prime}$ represents $H_{n} V_{1-} \rightarrow H_{n} W$ with respect to the bases $\left\{e_{i}\right\}$ and $\left\{f_{i}\right\}$. All the maps here are induced by inclusions. Finally, let $C_{1}$ and $C_{2}$ denote the matrices represent the maps $H_{n} W_{1} \rightarrow$ $H_{n} W$ and $H_{n} W_{2} \rightarrow H_{n} W$ with respect to the appropriate bases respectively. From $\left(^{*}\right)$, we have the following equation:

$$
B=C_{1} A,-\varepsilon B^{\prime}=C_{2}\left(-\varepsilon A^{\prime}\right), C_{1}\left(-\varepsilon A^{\prime}\right)=C_{2} A .
$$

These, together with the fact that $A+\varepsilon A^{\prime}=B+\varepsilon B^{\prime}=$ intersection form on $H_{n} V$, [5], give us the two equations in Lemma 2. Also, 
we have proved in [9] that both $A+\varepsilon A^{\prime}$ and $A-\varepsilon A^{\prime}$ are unimodular. Thus it follows from Lemma 2 that $B=C_{1} A=A\left(A-\varepsilon A^{\prime}\right)^{-1} A$.

Conversely, given a knot $\left(\Sigma^{2 n+1}, M^{2 n-1}\right)$ with its Seifert matrix $B$ satisfying the condition in Theorem 1 , we can construct a simple knot $\left(\Sigma_{1}, M\right)$ with an $(n-1)$-connected Seifert manifold $V$ and associ ated Seifert matrix $A$, [5]. Then we construct the 2-fold branched covering of $\left(\Sigma_{1}, M\right)$ to obtain a simple knot $\left(\Sigma_{2}, M\right)$ as in [4], [9], [12]. If we are in the p.l. category, then both $\Sigma$ and $\Sigma_{2}$ are the standard sphere $S^{2 n+1}$. Both $(\Sigma, M)$ and $\left(\Sigma_{2}, M\right)$ have the same Seifert matrix, hence they are actually equivalent, [8]. The involution $T$ is given by the covering translation for the branched covering.

4. Free involutions. Since the study of knots invariant under free involutions on spheres is very similar to that of knots fixed under involutions, [9], [10], the following theorem can be proved in a similar fashion.

TheOREM $1^{\prime} . \quad A$ simple knot $\left(\Sigma^{2 n+1}, M^{2 n-1}\right), n \geqq 3$, admits a free p.l. involution $T$ leaving $M$ invariant if and only if it has an associated Seifert matrix $B$ of the form $B=A\left(A-\varepsilon A^{\prime}\right)^{-1} A$ for some matrix $A$ with both $A+\varepsilon A^{\prime}$ and $A-\varepsilon A^{\prime}$ being unimodular.

5. The differentiable case. Let $T$ denote a differentiable involution on $\Sigma^{2 n+1}$ fixing $M^{2 n-1}, n \geqq 3$. We want to study the relation between the differentiable structure of $\Sigma$ and $\Sigma_{1}=\Sigma / T$. If $\Sigma_{1} \neq S^{2 n+1}$, then we may view $\left(\Sigma_{1}, M\right)$ as the connected sum of $\left(S^{2 n+1}, M\right)$ and $\Sigma_{1}$ along a disk disjoint from the seifert manifold $V$ and $M$. We then construct the 2-fold branched covering $\left(\Sigma_{3}, M\right)$ of $\left(S^{2 n+1}, M\right)$ with branched point set $M$. By the uniqueness of differentiable structure of the cyclic branched covering ([2] or [4]), it is easy to see that $\Sigma=2 \Sigma_{1}+\Sigma_{3}$, where the sum denotes the connected sum in the group of homotopy spheres $\Gamma_{2 n+1}$, [6].

In the case $n$ is odd, we let $\Sigma_{0}$ denote the generator of $b P_{4 k}=$ $\left\{y \in \Gamma_{4 k-1} \mid y\right.$ bounds parallelizable manifolds $\}$. Then we have the following proposition.

\section{Proposition 3. $\Sigma=1 / 8\left(\right.$ index $\left.\left(A+A^{\prime}\right)\right) \Sigma_{0}+2 \Sigma_{1}$.}

Proof. We first note that $A+A^{\prime}$ is a unimodular, symmetric, even matrix, hence its index is divided by 8, [6]. According to the remark in the preceeding paragraphs, we only have to determine the differentiable structure of $\Sigma_{3}$, the 2 -fold branched covering of $\left(S^{4 k-1}, M\right)$. 
We push the Seifert manifold $V$ into $D^{4 k}$, a disk having $S^{4 k-1}$ as its boundary; then use $V$ as the branched point set to construct a 2-fold branched covering $N$ of $D^{4 k}$ with $\partial N=\Sigma_{3}$, [4, $\S 4$. Proposition 5.6 in [4] tells us that the intersection form on $H_{2 k}(N)$ is given by $A+A^{\prime}$. All we have to do now is to show that $N$ is parallelizable. The Seifert manifold $V^{4 k-2}$ has the homotopy type of a wedge of $r$ copies of $S^{2 k-1}$, hence we may represent each of the basis element of $H_{2 k-1}(V)=r$ copies of $Z$ by an embedded $(2 k-1)$-sphere $S_{i}$. Each $S_{i}$ bounds a $2 k$-disk $D_{i}$ in $D^{4 k}$. Let $x$ denote the covering translation in the 2 -fold branched covering $N$ over $D^{4 k}$. Then $Q_{i}=x D_{i} \cup\left(-D_{i}\right)$ represent a basis for $H_{2 k}(N),[4$, p. 155]. $N$ has the homotopy type of a wedge of $r$ copies of $S^{2 k}$, represented by the $Q_{i}$ 's. Then the argument used in Lemma 4 (i) of [12] shows that the normal bundle of each $Q_{i}$ in $N$ is stably trivial. Thus $N$ is parallelizable, and it follows that $\Sigma_{3}=1 / 8\left(\right.$ index $\left.\left(A+A^{\prime}\right)\right) \Sigma_{0}$.

In particular, we see that $\Sigma_{0}$ does not admit an involution $T$ fixing a codimension 2 simple knot $M$ with $1 / 8\left(\right.$ index $\left.\left(A+A^{\prime}\right)\right)=$ even integer. In contrast, if $G$ is a free differentiable involution acting on $\Sigma^{4 k-1}$ leaving $M$ invariant, and $A$ a Seifert matrix for the equivariant knot complement $\left(\Sigma-M \times D^{2}\right) / G$; then we proved in [10] that $1 / 8$ (index $\left.\left(A+A^{\prime}\right)\right)=\sigma(G, \Sigma)=$ the Browder-Livesay index desuspension invariant, [11]. But we know that $\Sigma_{0}^{7}$, the generator of $b P_{8}$, admits a free involution $G$ with $\sigma\left(G, \Sigma_{0}^{7}\right)=0$, [3], [11, p. 63]. Thus $\left(G, \Sigma_{0}^{7}\right)$ admits an unknotted invariant $S^{5}$, [11], which implies 1/8(index $\left.\left(A+A^{\prime}\right)\right)=0$.

In the case $n$ is odd, we know that $b P_{4 k+2}=Z_{2}$ or $0,[6]$. Recall that $\Sigma_{1}=\Sigma / T$, where the involution $T$ fixes a simple knot $M$ in $\Sigma^{4 k+1}$. Then we have the following proposition.

\section{Proposition 4. $\Sigma=2 \Sigma_{1}$.}

Proof. As in Proposition 3, we only have to determine the differentiable structure of $\Sigma_{3}$, the 2-fold branched cover of $\left(S^{4 k+1}, M\right)$. The proof of Proposition 3 shows that $\Sigma_{3}$ bounds a $2 k$-connected parallelizable manifold $N^{4 k+2}$ with intersection form $A-A^{\prime}$. Then the argument in [5, p. 256-257] enables us to embed $N$ in $S^{4 k+3}$ in such a way that $\left(S^{4 k+3}, \Sigma_{3}\right)$ is a simple knot with Seifert manifold $N$ and Seifert matrix $A$ (see [4, p. 153] and [5, p. 256]). We know from [7, p. 544] that the Kervaire invariant of $N$ is the Arf invariant of $A$. Since $A+A^{\prime}$ is a symmetric, even, unimodular matrix, Lemma 2 in $[11$, p. 36$]$ shows that the Arf invariant of $A$ is zero. Hence $\Sigma_{3}$ is the standard sphere.

The author wishes to thank the referee for pointing out Proposition 4 to him. 


\section{REFERENCES}

1. S. Cappell and J. Shaneson, The codimension 2 placement problem and homology equivalent manifolds, Ann. of Math., 99 (1974), 277-348.

2. A. Durfee and L. Kauffman, Periodicity of branched cyclic covers, Math. Ann., 218 (1975), 157-174.

3. M. Hirsch and J. Milnor, Some curious involutions of spheres, Bull. Amer. Math. Soc., 70 (1964), 372-377.

4. L. Kauffman, Branched coverings, open books and knot periodicity, Topology, 13 (1974), 143-160.

5. M. A. Kervaire, Les noeuds de dimensions supérieures, Bull. Soc. Math. France, 93 (1965), 225-271.

6. M. A. Kervaire and J. Milnor, Groups of homotopy spheres, Ann. of Math., 77 (1963), 504-537.

7. J. Levine, Polynomial invariants of knots of codimension two, Ann. of Math., 84 (1966), 537-544.

8. An algebraic classification of some knots of codimension two, Comment. Math. Helv., 45 (1970), 185-198.

9. C. C. Liang, Semifree involutions on sphere knots, Michigan Math. J., 22 (1975), 161-163.

10. - Browder-Livesay index invariant and equivariant knots, Michigan Math. J., 23 (1976), 325-326.

11. S. López de Medrano, Involutions on Manifolds, Erge. der Math. Band, 59, SpringerVerlag, New York, 1971.

12. I. Tamura, Fixed point sets of differentiable periodic transformations on spheres, J. of the Faculty of Sci., Univ. of Tokyo, XVI (1969), 101-114.

Received January 1977. Partially supported by the University of Kansas General Research Fund.

UNIVERSITY OF KANSAS

LAWRENCE, KS 66045 



\section{PACIFIC JOURNAL OF MATHEMATICS}

EDITORS

RICHARD ARENS (Managing Editor)

University of California

Los Angeles, California 90024

C. W. Curtis

University of Oregon

Eugene, OR 97403

C. C. MOORE

J. DUGUNDJI

Department of Mathematics University of Southern Californa Los Angeles, California 90007

R. Finn AND J. Milgram Stanford University Stanford, California 94305

University of California

Berkeley, CA 94720

\section{ASSOCIATE EDITORS}

E. F. BeCKENBACH

B. H. NeUMANN

F. WOLF

K. YosHida

\section{SUPPORTING INSTITUTIONS}

UNIVERSITY OF BRITISH COLUMBIA UNIVERSITY OF SOUTHERN CALIFORNIA CALIFORNIA INSTITUTE OF TECHNOLOGY UNIVERSITY OF CALIFORNIA MONTANA STATE UNIVERSITY UNIVERSITY OF NEVADA, RENO STANFORD UNIVERSITY UNIVERSITY OF TOKYO UNIVERSITY OF UTAH NEW MEXICO STATE UNIVERSITY WASHINGTON STATE UNIVERSITY OREGON STATE UNIVERSITY UNIVERSITY OF WASHINGTON UNIVERSITY OF OREGON OSAKA UNIVERSITY 


\section{Pacific Journal of Mathematics \\ Vol. 73, No. 1 \\ March, 1977}

Thomas Robert Berger, Hall-Higman type theorems. $V \ldots \ldots \ldots \ldots \ldots \ldots \ldots$

Frank Peter Anthony Cass and Billy E. Rhoades, Mercerian theorems via

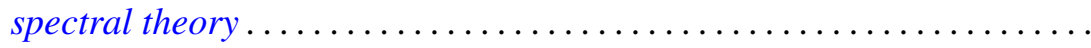

Morris Leroy Eaton and Michael David Perlman, Generating $\mathrm{O}(n)$ with

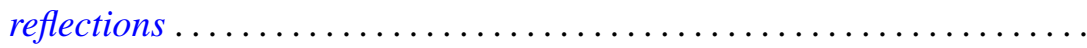

Frank John Forelli, Jr., A necessary condition on the extreme points of a

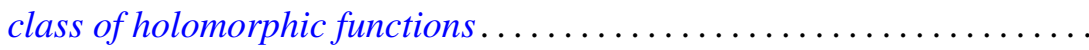

Melvin F. Janowitz, Complemented congruences on complemented

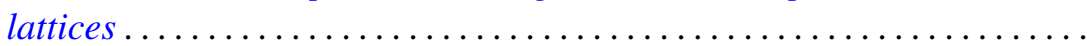

Maria M. Klawe, Semidirect product of semigroups in relation to amenability, cancellation properties, and strong $F \phi$ lner conditions....

Theodore Willis Laetsch, Normal cones, barrier cones, and the "spherical image" of convex surfaces in locally convex spaces ................

Chao-Chu Liang, Involutions fixing codimension two knots.............

Joyce Longman, On generalizations of alternative algebras .............

Giancarlo Mauceri, Square integrable representations and the Fourier

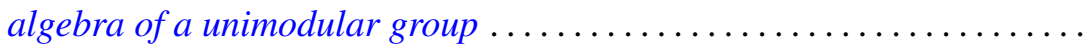

J. Marshall Osborn, Lie algebras with descending chain condition...

John Robert Quine, Jr., Tangent winding numbers and branched

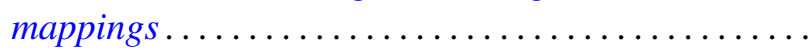

Louis Jackson Ratliff, Jr. and David Eugene Rush, Notes on ideal covers and associated primes .

H. B. Reiter and N. Stavrakas, On the compactness of the hyperspace of faces.

Walter Roth, A general Rudin-Carlson theorem in Banach-spaces ..

Mark Andrew Smith, Products of Banach spaces that are uniformly rotund in every direction.

Roger R. Smith, The R-Borel structure on a Choquet simplex ...

Gerald Stoller, The convergence-preserving rearrangements of real infinite series. ...

Graham H. Toomer, Generalized homotopy excision theorems modulo a Serre class of nilpotent groups...

Norris Freeman Weaver, Dehn's construction and the Poincaré conjecture....

Steven Howard Weintraub, Topological realization of equivariant intersection forms... 